\title{
Simplified Programming of Re-usable Skills on a Safe Industrial Robot - Prototype and Evaluation
}

\author{
Maj Stenmark \\ Dept. of Computer Science \\ Lund University \\ Lund, Sweden \\ maj.stenmark@cs.lth.se
}

\author{
Mathias Haage \\ Dept. of Computer Science \\ Lund University \\ Lund, Sweden \\ mathias.haage@cs.Ith.se
}

\author{
Elin Anna Topp \\ Dept. of Computer Science \\ Lund University \\ Lund, Sweden \\ elin_anna.topp@cs.Ith.se
}

\begin{abstract}
This paper presents a study on iconic programming support for mainly position-based lead-through programming of an ABB YuMi collaborative robot. A prototype tool supporting a hybrid programming and execution mode was developed and evaluated with 21 non-expert users with varying programming and robotics experience. We also present a comparison of the programming times for an expert robot programmer using traditional tools versus the new tool. The expert programmed the same tasks in $1 / 5$ of the time compared to traditional tools and the non-experts were able to program and debug a LEGO building task using the robot within 30 minutes.
\end{abstract}

\section{Keywords}

Kinesthetic teaching, User Study, Intuitive Programming of Industrial Robots

\section{INTRODUCTION}

In recent years, we have had a renaissance in industrial robotics: a new segment of robot models (e.g., UR5, ABB YuMi, Rethink Robotics' Baxter and KUKA's LBR iiwa) intended for safe human-robot collaboration and simpler robot programming have emerged.

The application areas for these robot models are typically assembly of consumer electronics or repackaging of (small) products. One difference between these robots and previous models is the lead-through programming, or kinesthetic teaching, mode. In this mode, the user can guide the robot arm into the desired positions and thus record and replay a robot program.

Kinesthetic teaching using force-guided motions [22], gravity compensation and variable stiffness [40, 39] support also non-expert operators to program and configure tasks [31], however, dynamic environments, uncertainty and the use of noisy sensor feedback are still challenges for the operator [34], and so far, experts are still needed to program the robots on the necessary levels of robustness and precision.

Permission to make digital or hard copies of part or all of this work for personal or classroom use is granted without fee provided that copies are not made or distributed for profit or commercial advantage and that copies bear this notice and the full citation on the first page. Copyrights for third-party components of this work must be honored. For all other uses, contact the owner/author(s).

HRI '17 March 06-09, 2017, Vienna, Austria

(C) 2017 Copyright held by the owner/author(s).

ACM ISBN 978-1-4503-4336-7/17/03.

DOI: http://dx.doi.org/10.1145/2909824.3020227
Programming by demonstration, $\mathrm{PbD}$, or learning from demonstration, LfD [7], is a well-established concept in service robotics, however, these methods often require multiple demonstrations which we consider less suitable in an industrial setting where rapid instruction and evaluation is needed. The desired approach in an industrial context is rather a one-shot demonstration, from which enough parameters for an executable representation can be extracted.

Orthogonal to the $\mathrm{PbD}$ approach is the use of high-level instructions to simplify robot programming for industrial tasks, e.g., using natural language [33] and semantically defined parameterized motion primitives [9], often called skills [28, 29]. Such semantic descriptions are desirable because they enable the automatic generation of, e.g., standard $\mathrm{PDDL}^{1}$-descriptions for planning and scheduling of tasks or path planning and generation from virtual models [30].

We propose to aim for a middle ground by combining programming by demonstration and parametrized skill representations to simplify robot programming, i.e., it must be possible for non-expert users to create their own skills from scratch and specify object references that can later be used as basic building blocks in high-level approaches.

In this paper we present a prototype of a programming tool for industrial robots, which enables non-expert users to program and edit simple industrial tasks using one-shot demonstrations, i.e., kinesthetic teaching, and also reduces the programming and debugging time for an expert programmer. We extracted initial requirements regarding functionality and behavior for the design of this tool from two case studies and evaluated it in two experiments with an expert user and in a study with 21 non-experts.

The remainder of the paper is organized as follows. In Section 2 we present the background and related work for our efforts. We present the case studies for extraction of requirements in Section 3 and our resulting prototype implementation in Section 4. In Section 5 we report on the evaluation of the tool through a user study with 21 subjects and by two expert experiments. We conclude the paper in Section 6 with a discussion of our findings and suggestions for future work.

\section{RELATED WORK}

The research into intuitive human-robot interaction and programming is so far more active in the field of service robotics, since service robots act in dynamic non-structured environments and are intended to interact with non-expert

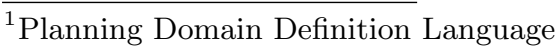


operators. Some of the following approaches are nonetheless relevant also for work in intuitive programming of industrial robots.

For instance, robots can learn from humans by observing the human carrying out a task and extract action parameters and objects involved in this process. Billard et al. use objects and locations as task parameters and can extract these in a new workspace from human demonstrations [7]. Feniello et al. synthesize the program specification for repositioning tasks from examples, e.g., their system can learn a program sequence that sorts objects by color [10]. Visuospatial skill learning is also used by Ahmadzadeh et al. [5] to extract the geometric constraints of the objects in a task and to learn the sequence of push and pull skills in a task. Using Gaussian mixture regression, trajectories can be generated from the learning results [13]. From multiple demonstrations, highlevel semantics such as intentions [11], transition conditions [17] and precedence constraints for sequencing of manipulation primitives $[19,21]$ can be extracted. Niekum et al. segment demonstrations automatically using (beta process autoregressive) Hidden Markov Models, learn the reference coordinates for each trajectory from clustering the demonstrations in different coordinate frames and iteratively refine the resulting finite state machine based on new demonstrations [25].

Low-level skills can be encoded as dynamic movement primitives (DMPs) [15] demonstrated using, e.g., Imitation Learning and further optimized using Reinforcement Learning. Examples of research in imitation learning methods for robot skill learning are given by Kormushev et al., who combine kinesthetic learning of positional trajectories with force profiles demonstrated using a haptic input device to demonstrate ironing skills [18]. Stenmetz et al. use an external force/torque sensor mounted on the robot arm to record force and position simultaneously and learn the force profiles for a PID-controller. Abu-Dakka et al. redeploy pegin-hole skills in new settings by adding exception strategies that randomly search for the hole to improve robustness [4]. Nemec et al. use a priori knowledge and reinforcement learning to reduce the number of demonstrations needed to teach a flip task to a robot [24] and Pastor et al. use reinforcement learning to optimize a pool strike [27]. All these methods, however, focus on one specific type of movement or task, each of which requiring a specific setup. Additionally, data driven approaches require multiple demonstrations with enough feature variation to extract task parameters and specifically for reinforcement learning based approaches it is a challenging problem to design a good cost function (which needs to be either taught or manually tuned).

Multiple demonstrations are time consuming, hence a oneshot approach is desired. Also, industrial tasks often require high precision, which can be difficult to achieve from generalizations between multiple demonstrations and the underlying representation of instructions must support adaption and debugging. Ko et al. list and motivate the main qualitative aspects of a programming (by demonstration) system that have to be evaluated, namely: accuracy and repeatability, adaptability and generality, learning fatigue, system complexity and production speed [16] .

Tsarouchi et al. identify various challenges in the humanrobot interaction for industrial robots, and while e.g., multimodal communication and learning by demonstration have received significant research attention also in this field, the user interfaces are often too complex for non-expert users [38]. The learning curve can be leveled by using visual programming environments, e.g., Huang et al. showed that a Scratch-like programming language reduced the programming effort [14] and Glas et al. have developed Composer for designing social robot behavior [12]. An increasing number of industrial robots provide template skills for common applications, e.g., the UR-series from Universal Robots as well as Baxter and Sawyer from Rethink Robotics come with template pick-and-place skills, and the Franka robot from Emika lets the user download expert made apps (i.e., skills) and combine these into more complex task using visual programming. Apps that can be used out-of-the box with little effort make these types of robots attractive, e.g., for small companies, however, programming new re-usable skills from scratch is still a challenge, which we try to handle with the interface proposed in this paper.

In order to bootstrap the instruction process, the interaction can be improved by systems that ask leading questions $[8,23]$, or by using other modalities such as speech, see, e.g., Pardowitzet al. [26] or a graphical user interface so that the task constraints generated from a single demonstration can be understood and edited by the operators [6, 20]. We plan to enhance our system with the necessary reasoning capabilities so that such an approach to supporting the user can be integrated.

\section{CASE STUDIES}

We analyzed the programming process and required motion types and primitives from two rather complex applications that we had previously built for the dual-arm ABB robot YuMi. One is an emergency stop button assembly that uses force-based motions for snapping and screwing together switches and nuts $[36,2]$. The second case is a position-based gift-wrapping application, which was developed as part of a commercial campaign in Sweden during the fall of 2015 and toured consumer electronic retail stores wrapping gifts all over Sweden [3, 34, 37].

Both applications rely on synchronized motion between the two arms of the ABB YuMi dual-arm robot. The robot is designed for safe human-robot collaboration and each arm has seven degrees of freedom. These features are used in the gift-wrapping application since the wrapping process uses a collaborative step and multiple contact points on the arm. Videos of both applications are available through YouTube $[2,3]$.

We identified several issues during the programming process, which are mostly related to the traditional programming interfaces and assumed workflow for the YuMi [34, 37]. From those issues, we derived requirements for the development of a programming interface that supports (and is supported by) our underlying architecture for skill representation and robot code generation [32].

\subsection{Traditional interfaces and interaction}

The ABB robot can be programmed in the ABB programming language RAPID either using an engineering tool called RobotStudio, a Windows Universal App (YuMi Online) or by using the teach pendant (an integrated joystick and touch display interface for direct robot control, a standard piece of equipment usually accompanying industrial robots, see Figure 1), directly. RobotStudio and the app can run on the same Windows device and are connected to 


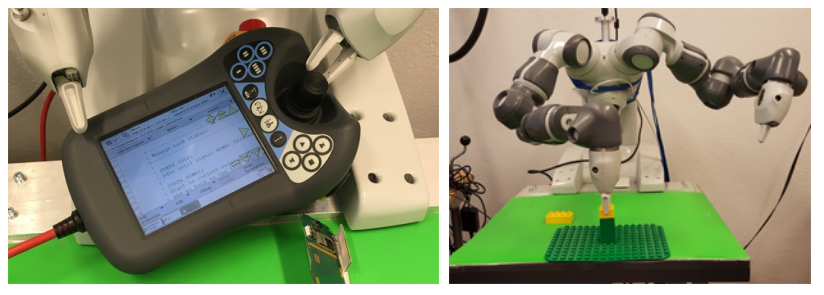

Figure 1: ABB's (YuMi's) teach pendant (left) and YuMi in the LEGO building task (right).

the robot through a local network. Each of the tools has different features. RobotStudio is intended for editing RAPID programs and for simulation. The app has an online programming interface supporting a few commands (motions and gripper actions) and has a limited debugging support. It has however a kinesthetic programming mode, lead-through, which makes it easy to teach positions in free space. Contact situations are more suitable to teach with the joystick on the teach pendant since contact forces will move the arms out of position if lead-through is enabled. The teach pendant can also be used for debugging, for example by moving the program pointer and executing instructions one-by-one.

The traditional workflow starts with offline programming and simulation before deploying a program on the physical robot. First, the program can be written and executed in the virtual environment in RobotStudio. When the user is satisfied, the program is deployed on the physical system and testing in the real environment can begin. User interaction can be added into the RAPID program by inserting dialogue prompts into the program. Then the resulting program can be deployed in the factory and the robot operator will only interact with the robot using the dialogues. All error handling procedures need to be added beforehand by the programmer.

It is not trivial to include (contact) forces and deformable material into simulations, hence, our applications required online programming, i.e., testing and updating using the physical robot system including the lead-through functionality for kinesthetic teaching of poses, which was non-trivial with the given interfaces due to their different features. Specifically, in the gift-wrapping application the programmers were required to sometimes circumvent the safety mechanisms originally designed for unsafe robots, but still deployed on the inherently safe YuMi.

\subsection{Analysis}

Our observations from programming the two applications provided an initial list of system requirements regarding which basic instructions should be possible to rapidly include and update in a program and some hypotheses about better user interaction and support, which we explain briefly in the following.

Actions: The applications required mostly motions specified through gripper target positions in Cartesian space (both relative specified objects or in world coordinated). Secondly, joint configurations were often used in the giftwrapping application for specifying the position of other parts of the arm than the gripper. Also, both applications relied on contact motions (i.e., motions that stop when a force threshold is reached) for robust task execution. Other actions identified as common are gripper commands (open and close), movement to synchronization points for both arms, and synchronized motions of both arms.

Reuse, refinement and update: In order to be able to reuse a task, i.e., a program sequence with small changes, e.g., in object positions, it must be possible to specify work objects so that motion primitives can be programmed using relative reference coordinate systems. Also, it must be possible to easily change and adapt reference coordinate systems for existing work pieces, as it must be assumed that even expert users occasionally forget to specify the correct reference immediately.

User interaction: In the two applications, user interaction as part of the normal operation was limited to handling discrete events in the program flow. For stopping or pausing the execution, the operator touched the robot and simply triggered the safety system break, but that also required manual restart of the robot and required rather long cycles to update and test specific instructions. Here, we assume that a rapid cycle of stopping the execution, changing and updating instructions where necessary and testing (executing again) is desirable.

Compatibility: Since we initially assume only a subset of instructions (actions as described above) to be available in our graphical user interface, the interface should generate an editable program file that an expert user can refine further by using the traditional tools and interfaces.

Based on this list of observations and assumed requirements, we designed a prototype interface supporting users in quickly creating basic programs (skills) through kinesthetic teaching and iconic programming [35]. These skills can then later be refined and form the basis for more complex programs, which can be generated using even more high-level approaches to robot programming, as proposed earlier [33].

\section{IMPLEMENTATION}

In the following we describe the graphical user interface, which we developed based on the previously described findings from our case studies. The interface was also meant to be a tool for investigating, how our underlying ideas, mainly the skill representation and the reuse of skills through parameterization, would support non-expert users in programming an industrial robot.

A screen capture of the implemented programming tool is shown in Fig. 2. It is implemented as a touch-enabled Windows app running on a Surface Pro with 8 GB RAM and a wireless connection to the robot controller. The implementation is programmed in $\mathrm{C} \#$ and uses $\mathrm{ABB}$ robot controller libraries for network communication and math calculations.

\subsection{Features}

The graphical interface divides the screen into an instruction panel to the left and a program panel to the right. In the upper left corner, the built-in basic actions are displayed in yellow, while the user-created skills (sequences and combinations of such primitives or other skills) are added to the list in purple. The default actions are the following:

Move: a fine-point slow move. When this instruction is added to the robot program sequence, it takes the current position of the active arm and saves it as precise goal position (trajectories between specified points are generated by the robot 
controller so far). The target is saved with three positions: the exact joint position for the robot arm, the Cartesian position relative to the world (or robot base) system and, if an object is selected, the position relative to the object. Thus, the user can switch coordinate systems between objects or to absolute positions, or between Cartesian and joint space motions without updating the position.

Via: a fast motion with zone parameter $5 \mathrm{~mm}$ as default, i.e., getting close to the specified pose within this zone is considered sufficient. Analogously to the fine-point move, the position is saved in absolute and relative coordinates as well as in joint space.

Open and Close for fully opening and closing the gripper. The gripper action will execute when added to the program.

Contact Move: a movement towards a contact point which will stop when detecting an estimated contact force. The current position of the robot will be used to set a contact position, but the motion will continue beyond that point.

Sync: adds synchronization points, i.e., points in the program sequence where both arms should reach the specified pose at the same time.

Fingers: an action for setting the finger positions exactly and changing the gripping force of the fingers.

Locate objects: will call a local procedure on the robot controller for detecting objects using the built-in cameras on the robot's hands. For the time being, the camera calibration, image settings and detection algorithms must be provided by an expert and reloaded into the controller. The detected object's reference system will, however, be updated each time the procedure is executed hence the user can use the object references to program the robot without considering the underlying code.

Under the actions, the user can add coordinate frames related to objects by pointing with the robot. These can be updated when the object is moved.

The current robot programs for respective arms are displayed to the right. Each action has a line with an individual play arrow, an editable name, a color label for the relative coordinate system, a button for updating the position, editing and a picture. The order of the sequence can be edited by dragging and dropping.

Additional buttons provide functionality for opening and closing the grippers without adding program instructions, executing, toggling the lead-through functionality of the robot and saving/loading a complete workspace consisting of skills, objects and current robot program.

\subsection{Reuse of skills}

Action (sub)sequences can be selected and stored as skills, these will show up in the action list as purple buttons. In the sequences the skills can be expanded and edited further.

The user can add skills with single instructions but with application-specific parameters such as speed, zone (precision parameter for movement), and object data, and thus have for example multiple types of parameterized motions. E.g., in the gift-wrapping application, the speed was different for motions manipulating the paper and pushing the box, hence, each time an instruction was added the speed parameter had to be set to the correct value.

The actions and skills are robot agnostic and can be switched between the arms. The Sync actions can of course cause problems if not moved in pairs. If a skill was programmed using one arm, the joint positions cannot be reused for the other arm, so the default execution will use the Cartesian positions either relative to the object or in absolute coordinates. By selecting multiple actions, the relative object can be changed and updated to another by one button click, recalculating the relative offset to the new object using the absolute coordinates system.

Once the user requests a sequence to be executed (played), the high-level representation of the program is used to generate low-level instructions (RAPID code) for the robot controller currently connected to the user interface.

Our current prototype implementation does not (yet) support more advanced (and natural) force-based motions or logging and executing trajectories, however, a respective extension is subject to ongoing work.

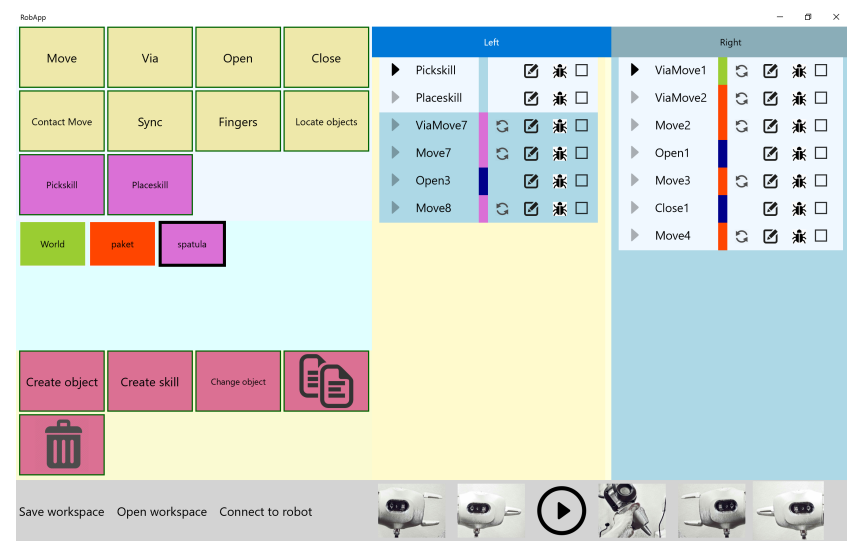

Figure 2: The graphical user interface.

\subsection{User interaction and interference}

The programming and debugging modes are merged into one hybrid mode, where the speed can be reduced during debugging and the safety system stops the robot in case of collisions with objects or the user, or predicted self-collisions.

The programming interface is designed to be forgiving towards the user. The user can program the entire sequence in absolute coordinates and then later add objects to the workspace and change the reference of the motion without reprogramming. The actions can be tested, refined, and updated individually, and any type of sequence can be saved as a skill.

Debugging is not a separate part of the execution process; however, the current implementation only supports running of individual actions and skills, or the entire sequence. Future work includes additional running and debugging options, e.g., running only a selected subset of actions, starting the execution at arbitrary points in the program and toggling debugging break points. The existing tools for jogging the robot using a joy stick or editing the generated RAPID program in a text editor are still available.

\section{EVALUATION AND USER STUDY}

The programming interface was evaluated by comparing programming times for one expert who programmed two robot tasks using both the new interface and traditional programming methods and through a user study with 21 
non-expert, although technically or mathematically interested and experienced, users in a LEGO building task.

We see this study as an opportunity to observe potential users interacting with an industrial robot in a realistic setting, as the LEGO building task, although seemingly a child's play, has quite some challenges similar to those of assembling smaller products like, e.g., mobile phones. In the following paragraphs, we describe the study setup which had the original background of testing the effects of skill re-use and parameterization for non-expert programming of assembly tasks for industrial robots [35]. We will thus in the following explain the different conditions for testing our hypotheses on the re-use of skills and break down our results by these conditions, but we had to realize that the study turned out to be as much an evaluation of the interface's general applicability as it was a tool for testing our original hypotheses. Thus, we focus on the rather general results about the overall benefits of our approach for non-expert programming of industrial robots and on the results we could achieve in the experiments carried out by the expert.

\subsection{Study setup}

We recruited our subjects mainly from an undergraduate (introductory) course on robotics and through advertising at the departments of Computer Science and Automatic Control at our university to ensure a somewhat technical or software oriented background. Here, we assume that this would be the case also for prospective users (instructors) of this type of robots in industrial contexts. However, we had the subjects fill in a questionnaire to also collect data about how they ranked their own experience with programming and technical equipment, to get a more accurate understanding regarding their background.

Our subjects were asked to program one arm on a dualarm ABB YuMi with standard grippers to assemble different types of LEGO Duplo bricks using the robot's lead-through mechanism for kinesthetic teaching of poses in combination with our graphical interface for iconic programming. Figure 1 shows the setup for the study with the robot just finishing one step of the overall task. Routines for locating two specific types of LEGO bricks with the help of the builtin camera of the robot gripper were already provided by the experiment leaders as functionalities to be invoked by inserting a respective instruction in the program sequence. This was done for convenience as the camera calibration procedure is quite tedious and time consuming and should not be part of the study. The LEGO piece localization procedures had the arm move to a predefined position, from where the pieces could be localized robustly, given that they were placed within a coarsely sketched area. This allowed to specify pick-up poses relative to the work piece. We are aware that this is quite sophisticated knowledge about robotic systems to be handled for non-experts, however, it gave us the opportunity to observe how our subjects could be supported by our interface in switching between reference coordinate frames for the different objects involved in the task.

\subsubsection{Conditions and specific tasks}

The overall task was carried out in two phases: The first phase was the same for all participants while the second phase divided the users evenly into three groups corresponding to the three different test conditions. During the first phase, the instructions were to program the robot arm to pick up a LEGO Duplo "2x2" piece (referred to as "small LEGO") and insert it on top of another small piece, which was already the top of a little "tower" (task step 1). This program should then be used to create a skill, i.e., a re-usable representation of a sequence of motion primitives. For the second phase, the participants were asked to program the robot arm to carry out the same task another three times, but with a "2x4" piece ("large LEGO"), which should be placed first "centered" (step 2), then "hanging over on the left" (step 3), and finally "hanging over on the right" (step 4) with respect to the "tower top". For these three steps, the different conditions defined the programming paradigm, i.e., the first group, Group A, was to re-use and refine their own previously created skills, Group B was to re-use an expertmade skill and Group $\mathrm{C}$ was a control group in which participants should program each step from scratch without re-use of previously created and saved skills.

The instructions were given in written form, for each phase separately. After reading the instructions for step 1 the participants were introduced to the robot and the programming interface by one of the experiment leaders for a couple of minutes and then started to work on their tasks. The experiment leader observed the process, both to be able to resolve technical problems (the robot stops and needs to be reset when it collides with any obstacle) and to assist the subjects with the handling of the programming tool in case they needed help (the tool has no built-in "help" functionality so far). Upon completion of step 1 and if the time frame allowed, the participants received the instructions for steps 2-4.

From pilot testing with involved researchers we had estimated that roughly 30 minutes for the actual programming was a suitable time limitation for each participant, however, this had the effect that in quite some cases the participants would not reach far enough into the second phase to complete all four programming steps. In this paper, we will thus focus on rather general evaluation criteria like success rate and time needed for completion in the first phase, and how many further steps the subjects managed to complete before running out of time. A more thorough analysis regarding the effect of the different methods and the subjects' individual ways of handling the task steps of the second phase is planned as future work.

After either completing all four steps or running out of time, we asked our subjects to fill in a questionnaire with both demographic questions (gender, occupation) and several five-level scale questions ranging from "not at all" to "absolutely" about their previous experiences with robots or programming, and the level of comfort they felt when handling the robot. Additionally, there was one yes/no question on whether they had ever programmed a physical robot before. We also gave them the opportunity to explain in free text what they found most difficult and what they would suggest to be changed in the programming interface.

\subsubsection{Analysis}

We had 21 participants (five female, 16 male), seven in each of the condition groups A, B, and C respectively. Figure 3 shows the distribution of professional background and gender as well as whether they had any previous experience of robot programming. The two participants with a non-technical professional background registered as communicator and nurse / kindergarten teacher respectively. 

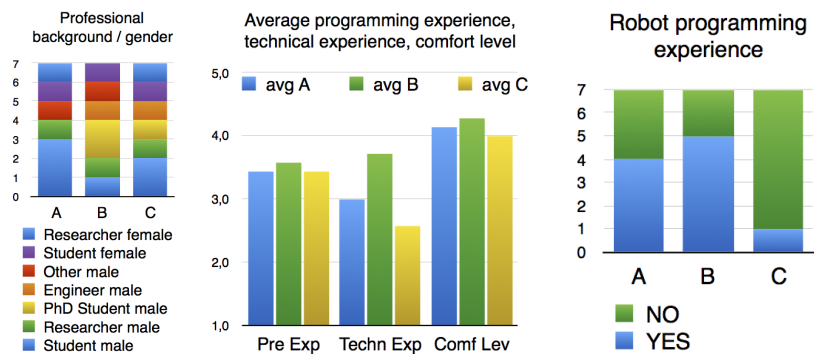

Figure 3: Participants' background and gender (left), experience and comfort level ratings (middle), and experience in programming a robot (right).

Table 1: Success (overall rating average) and time (minutes average)

\begin{tabular}{|l|l|l|l|l|l|}
\hline & Success & $\begin{array}{l}\text { Time } \\
\text { Step 1 }\end{array}$ & $\begin{array}{l}\text { Time } \\
\text { Step 2 }\end{array}$ & $\begin{array}{l}\text { Time } \\
\text { Step 3 }\end{array}$ & $\begin{array}{l}\text { Time } \\
\text { Step 4 }\end{array}$ \\
\hline \hline All & 6.0 & 20.6 & 8.1 & 5.0 & 3.5 \\
\hline Group A & 5.0 & 20.4 & 14.5 & 1.5 & - \\
\hline Group B & 9.0 & 18.4 & 3.0 & 5.5 & 2.0 \\
\hline Group C & 4.14 & 22.9 & 9.5 & 9.0 & 8.0 \\
\hline
\end{tabular}

We evaluated the trials for each participant according to the following criteria:

time step $n$ : the time spent on completing step $n$ after completing the previous step.

success rate: the sum of the success ratings for each step $n$ according to the following criteria full (3) - the LEGO piece was fully attached on top of the "tower" robustly by the robot in step $n$; partial (2) - the LEGO piece was placed correctly but not (entirely) attached; fail (1) - the step was not carried out fully, i.e., the LEGO piece was not placed correctly; $n / a(0)$ - the step was not carried out at all.

Table 1 summarizes the (average) results according to these criteria, broken down by the different groups, while Table 2 shows the number of subjects managing the respective step with at least partial success.

We can state that almost all participants managed to handle the first step with at least partial success. The two participants (group C) who failed completing the first step had difficulties in understanding how the robot would move (directly between specified poses) after their instructions.

One of them stated also to have felt rather uncomfortable (2) when handling the robot, despite marking rather high levels of programming (5) and technical (4) experience, and mentioned the two coordinate systems as difficult to understand. This low comfort rating was in fact the only one below the "neutral" rating of 3 for all 21 participants. Worth noting is also that all three participants reaching full success in step 4 belonged to group B, i.e., the condition with the task to re-use an expert-made skill for the last three steps. As step 2 for this group was mainly to analyze and understand the provided skill, this obviously saved them a lot of time compared to the other groups. Overall we can state,
Table 2: The number of subjects reaching success rating 3-2-1 respectively in the different steps.

\begin{tabular}{|l|l|l|l|l|}
\hline & $\begin{array}{l}\text { Step 1 } \\
3-2-1\end{array}$ & $\begin{array}{l}\text { Step 2 } \\
3-2-1\end{array}$ & $\begin{array}{l}\text { Step 3 } \\
3-2-1\end{array}$ & $\begin{array}{l}\text { Step 4 } \\
3-2-1\end{array}$ \\
\hline \hline All & $13-6-2$ & $11-3-0$ & $7-2-0$ & $3-0-1$ \\
\hline Group A & $5-2-0$ & $3-1-0$ & $1-1-0$ & $0-0-0$ \\
\hline Group B & $5-2-0$ & $6-0-0$ & $5-1-0$ & $3-0-0$ \\
\hline Group C & $3-2-2$ & $2-2-0$ & $1-0-0$ & $0-0-1$ \\
\hline
\end{tabular}

that 14 of 21 participants managed to understand the tool, the robot's movements, and the LEGO bricks' properties (including the challenge of robustly attaching them to each other) sufficiently well to complete two steps of the overall tasks within 30 minutes.

As we noted a somewhat uneven distribution of programming and technical experiences over the different groups, we checked for correlation effects between experience ratings and success rates or time needed for the different steps respectively, but we could not find any obvious connections; programming experience, technical experience and robot programming experience had all a very low negative correlation $(-0.18,-0.18$, and -0.23$)$ with time for completion of step 1 and a slightly stronger, but still very low, positive correlation $(0.24,0.22$, and 0.33$)$ with the overall success rate. Thus, we consider the different outcomes for the groups as an indication for an effect of the different conditions, i.e., being able to reuse previously programmed or even provided skill representations simplifies the programming process significantly, as the subjects in the control group (C) needed roughly the same amount of time for each step in the second phase, when programming from scratch.

In group $\mathrm{A}$, who should re-use their own previously created step1 skill for the following steps, the subjects needed some time to handle the updating and parametrization of the skill in step 2, but once they had understood how to do this, the following steps were handled within rather short time frames, which were even shorter than those for group $\mathrm{B}$, who otherwise had very high success rates, but had to understand and update a program that they were not familiar with from the beginning. However, sample sizes are small and became even smaller over the different steps, hence, these results should be seen with some caution and treated as trend indications rather than definite findings.

\subsubsection{Specific challenges and observations}

As indicated above, all participants but one felt at least neutral, if not comfortable, when handling and programming the robot. We consider this a confirmation for the overall idea of combining kinesthetic teaching with iconic programming in an interactive way, i.e., allowing for frequent switches between execution, testing / debugging and further instruction, is a good starting point for further development of the tool. However, we also have to see the comments (and observations) regarding the challenges the subjects experienced, which we summarize in the following, according to how often they were mentioned (high frequency first). 
Precise positioning and fixating of the LEGO piece Many subjects mentioned the placement of the piece as a challenge. In fact, attaching two LEGO bricks to each other robustly with slippery robot grippers is tricky, and the success seems to depend a lot on whether the respective subject could determine a good strategy from the LEGO properties (the piece clicks best into place when being "folded" and then pressed, rather than through hovering over the target and moving straight down at once). The expert-made skill provided for group B followed this strategy, and worked very robustly (it merely took the expert-one of the researchers involved in the study setup - roughly two minutes to create this skill). Here, we plan to equip a future high-level interface for programming with the capability of providing suggestions based on previously known skills for similar work pieces to find the optimal strategy for a given work piece.

Different reference coordinate frames and switching between them As mentioned before, we are aware that selecting relevant coordinate frames is quite a challenge for any non-expert user, however, we can now, based on our observations of how the subjects managed to solve the problem and how exactly they could be supported, design further support based on reasoning and mixed-initiative interaction into future tools. We also observed, that many subjects simply forgot to assign the correct coordinate system, even though they understood how relative motions work. This was anticipated in the design of the tool, allowing the user to switch reference frames for an already existing movement, which made it very easy to correct the program after an issue with the reference frames was discovered.

Understanding all functionalities of the tool in the limited time frame, handling the interface This suggests that we might even have had better results, had the subjects been given more time to "play" with the tool. Some stated also informally after finishing, that "now, it would be great to start over, now I have understood this!".

Pose recording vs trajectory recording, understanding robot movement Some of the subjects, as also mentioned above, struggled to understand the concept of specifying positions to move to rather than trajectories to move along. In particular, the "contact move" posed a challenge as it results in a movement towards a virtual projection of a desired pose beyond the contact point. Further development of respective tools to include trajectory and contact force profile teaching is ongoing work.

Robustness, compensating for the LEGO piece sometimes slipping from the gripper As we did not make use of specifically designed grippers for LEGO-bricks, this was obviously a problem in some cases. It could be compensated for only through a good picking and placing strategy that would be robust to the slipping. Using more appropriate tools on the robot for grasping specific work pieces is obviously a good strategy, and is subject to investigations within the project work for the ongoing SARAFun project [1].

We see quite some of the challenges pointed out by our subjects as concrete confirmation of assumptions we had regarding obvious points to work with in the future.

In addition to not finding a strong correlation between experience and success rates, we observed that the one person with the least technical background not only felt very com-
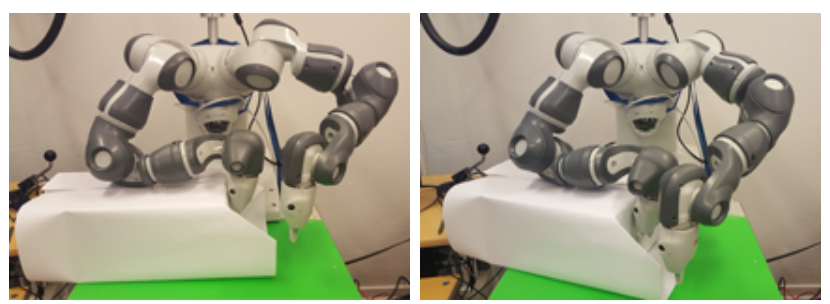

Figure 4: Paper folding (left). When rotating the box, only two positions must be updated (right).

fortable (4) when programming the robot, but also managed to complete all four steps of both phases within 21 minutes with full success. On the other hand, we observed highly experienced $\mathrm{PhD}$ students in Automatic Control struggling with the task for significantly longer periods of time and had one participant with high experience ratings failing in the first step already.

In a sideline of the study, we had one nine-year old child test the interface in the study setup. The child, while certainly an expert in LEGO building, was not a proper study participant due to a lack of English reading capabilities, but managed with only a little help regarding the switch of reference coordinate frames and the use of the contact move to program the first step (picking up and inserting the $2 \times 2$ piece on top of the "tower") within 20 minutes.

Boldly speaking, these observations seem to suggest that understanding of the task and assuming the robot to follow kinesthetic instructions as precisely as a (fellow, smaller) child, rather than assuming it being capable of understanding complex instructions, made it easier for the subjects to solve the task.

In the following we will present the evaluation of the programming tool carried out by an expert user, before drawing conclusions from our findings.

\subsection{Expert evaluation}

One of the case studies we presented in section 3, the giftwrapping task, formed the basis for the evaluation of the programming tool by an expert who is quite familiar both with programming YuMi in the traditional way using a teach pendant and coding directly in RAPID as well as with the specifics of the gift-wrapping process. For the evaluation, a folding task was chosen, where the right arm fixated a gift box with the elbow while the left straightened the corners using nine positions (fine and via), as illustrated in Figure 4. The operator used the programming tool presented in this paper to add a packet object to the world and programmed the actions for the left arm relative to this packet object. Then the box was moved to a different position and the packet and right arm positions were updated accordingly. After that the left-hand program executed without change. The entire programming, involving one test execution for each position took slightly below two minutes. The same process was then programmed using traditional tools which took slightly more than 11 minutes.

We also evaluated the interface in another context. Here, the overall task was to create two skills, one for picking a tool from a fixture and one for placing it back into the fixture. Creating a tool object and storing the positions for the picking and placing and saving these as skills took 70 seconds 
with our interface and more than 5 minutes with traditional programming methods, hence, the initial programming time was reduced by $80 \%$ using our interface compared to the traditionally provided methods. A more realistic scenario would then certainly involve multiple adjustments and parameter tuning, if required using the advanced settings in the traditional tools, however, executing and updating single instructions requires two button presses in our tool, while the traditional approach requires at least five steps, twice that if more than one coordinate system is used in the task.

We can thus state, that for an initial online programming process of applications that are difficult to test in a simulator (e.g., paper folding), our interface speeds up the programming process significantly. As it allows for debugging, executing, as well as updating and even reordering single steps in the robot program, we would expect our programming interface to be quite suitable (and faster than the traditional methods) also for more complex procedures.

\section{CONCLUSION}

In this paper we discussed our approach to high-level, intuitive programming of industrial robots for assembly tasks. We presented findings from an analysis of two case studies regarding the desired capabilities and functionalities of a programming interface for online programming in settings where the traditional work flow is not suitable, due to, e.g., simulation for testing not being an option. Based on these findings we developed a programming interface that supports a hybrid instruction and evaluation (execution) process and thus quick implementation and test cycles through a graphical interface for iconic programming. The interface supports also the specification of objects and their positions in the work cell of the robot, as well as updating these at any time. It is also possible to reorder instructions graphically and sequences of instructions can be saved as a skill for later re-use and refinement. We evaluated both with experts and 21 non-expert users in a user study and can state the following regarding the improvements and simplifications we assumed our tool to yield.

Already the basic support of a rapid instruction, test, and execution cycle with the possibility to reorder and update single instructions for refinement reduced the programming time for an expert by $80 \%$ compared to using the traditional programming tools and interfaces. This was specifically supported by the possibility to easily specify and refine object positions and reference frames and assigning them to certain instructions, so that re-use of program parts (skills) was enabled or even (as evaluated) a full program for one robot arm could be run without modifications, when the pose of the object the respective instructions referred to was updated. The possibility to change the frame of reference for already existing instructions is an additional convenience, as it is easy to forget to always set the correct one from the beginning, even for experts.

Our evaluation with 21 non-experts showed that the programming tool provides an interface sufficiently easy to handle, so that 19 of 21 participants could construct a satisfying solution to a LEGO-building task within 30 minutes, and 14 of them even managed to complete one or several follow-up steps. We can also state, that the reuse of previously created (own) skills and even more so of provided, expert-made skills, simplifies the programming process significantly. Our subjects struggled mainly with the precise positioning of the
LEGO-bricks so that they would click into place firmly and (as we previously assumed) with the different object reference frames needed to handle picking up the pieces from arbitrary poses. For the first issue, we assume that process knowledge (how to best approach one work piece with the other to attach them correctly) was here of more significance than knowledge about the robot system, as we could not find an obvious correlation between success rate and experience. For the reference coordinate system issue we can state that although the users - even if they had understood the problem with the frames - would frequently forget to assign the correct one to their instructions, they managed to correct their mistakes quickly with the functionality for changing reference frames.

Other tools for high-level programming often assume an existing library of robot skills, e.g., tools supporting natural language instructions as for example presented by Stenmark et al. [32] need basic knowledge of objects (and their coordinate frames) and that it is possible to update and refine solutions suggested by a learning and reasoning system to industrially adequate robustness and precision. Hence, we see our interface as appropriate to support even non-expert users in handling the construction of such basic knowledge items as well as in updating and refining existing solutions.

Overall, we see our implementation as a successful prototype, which we can now use as a basis for further development and studies and software distribution within the research community. The integration of high-level (natural language) instruction capabilities with the interface as well as functionalities for teaching force profiles and trajectories as basic movement primitives, or skills are subject to ongoing work. This would presumably support the user even more in teaching contact moves and specific operations like snap-fit assembly. As the reference coordinate problem is difficult for users to both understand and then also to remember in the programming process, we plan to integrate mixed-initiative support based on reasoning about possible options regarding the assignment of reference frames to instructions.

\section{ACKNOWLEDGMENTS}

The research leading to these results has received funding from the European Community's Framework Programme Horizon 2020 under grant agreement No 644938 SARAFun.

\section{REFERENCES}

[1] H2020 sarafun - smart assembly robots with advanced functionalities - grant agreement no 644938. http://h2020sarafun.eu/. Accessed: 2016-10-03.

[2] Robotic assembly of emergency stop buttons youtube video. https://youtu.be/7JgdbFW5mEg. Accessed: 2016-10-03.

[3] YuMi wraps Christmas gifts - youtube video. https://youtu.be/ASEtz2M1RiY. Accessed: 2016-10-03.

[4] F. J. Abu-Dakka, B. Nemec, A. Kramberger, A. G. Buch, N. Krüger, and A. Ude. Solving peg-in-hole tasks by human demonstration and exception strategies. Industrial Robot: An International Journal, 41(6):575-584, 2014. 
[5] S. Ahmadzadeh, A. Paikan, F. Mastrogiovanni, L. Natale, P. Kormushev, and D. Caldwell. Learning symbolic representations of actions from human demonstrations. pages 3801-3808. IEEE, 2015.

[6] S. Alexandrova, M. Cakmak, K. Hsiao, and L. Takayama. Robot programming by demonstration with interactive action visualizations. In Proceedings of Robotics: Science and Systems, Berkeley, USA, July 2014.

[7] A. Billard, S. Calinon, and R. Dillmann. Learning from humans. In Springer Handbook of Robotics, Chapter 74, pages 1995-2014. Springer, 2016.

[8] M. Cakmak and A. L. Thomaz. Designing robot learners that ask good questions. In Human-Robot Interaction (HRI), 2012 7th ACM/IEEE International Conference on, pages 17-24, March 2012.

[9] J. Felip, J. Laaksonen, A. Morales, and V. Kyrki. Manipulation primitives: A paradigm for abstraction and execution of grasping and manipulation tasks. Robot. Auton. Syst., 61(3):283-296, Mar. 2013.

[10] A. Feniello, H. Dang, and S. Birchfield. Program synthesis by examples for object repositioning tasks. In 2014 IEEE/RSJ International Conference on Intelligent Robots and Systems, pages 4428-4435, Sept 2014.

[11] B. Fonooni, T. Hellström, and L.-E. Janlert. Priming as a means to reduce ambiguity in learning from demonstration. International Journal of Social Robotics, 8(1):5-19, 2016.

[12] D. F. Glas, T. Kanda, and H. Ishiguro. Human-robot interaction design using interaction composer: Eight years of lessons learned. In The Eleventh $A C M / I E E E$ International Conference on Human Robot Interaction, HRI '16, pages 303-310, Piscataway, NJ, USA, 2016. IEEE Press.

[13] B. Huang, M. Li, R. L. Souza, J. J. Bryson, and A. Billard. A modular approach to learning manipulation strategies from human demonstration. Auton. Robots, 40(5):903-927, June 2016.

[14] J. Huang, T. Lau, and M. Cakmak. Design and evaluation of a rapid programming system for service robots. In 2016 11th ACM/IEEE International Conference on Human-Robot Interaction (HRI), pages 295-302, March 2016.

[15] A. J. Ijspeert, J. Nakanishi, and S. Schaal. Learning attractor landscapes for learning motor primitives. Technical report, 2002.

[16] W. K. H. Ko, Y. Wu, K. P. Tee, and J. Buchli. Towards industrial robot learning from demonstration. In Proceedings of the 3rd International Conference on Human-Agent Interaction, pages 235-238. ACM, 2015.

[17] J. Kober, M. Gienger, and J. J. Steil. Learning movement primitives for force interaction tasks. In Robotics and Automation (ICRA), 2015 IEEE International Conference on, pages 3192-3199. IEEE, 2015.

[18] P. Kormushev, S. Calinon, and D. G. Caldwell. Imitation learning of positional and force skills demonstrated via kinesthetic teaching and haptic input. Advanced Robotics, 25(5):581-603, 2011.

[19] A. Kramberger, R. Piltaver, B. Nemec, M. Gams, and A. Ude. Learning of assembly constraints by demonstration and active exploration. Industrial Robot: An International Journal, 43(5):524-534, 2016.

[20] A. Kurenkov, B. Akgun, and A. L. Thomaz. An evaluation of gui and kinesthetic teaching methods for constrained-keyframe skills. In 2015 IEEE/RSJ International Conference on Intelligent Robots and Systems (IROS), pages 3608-3613, Sept 2015.

[21] S. Manschitz, J. Kober, M. Gienger, and J. Peters. Learning to sequence movement primitives from demonstrations. In 2014 IEEE/RSJ International Conference on Intelligent Robots and Systems, pages 4414-4421, Sept 2014.

[22] D. Massa, M. Callegari, and C. Cristalli. Manual guidance for industrial robot programming. Industrial Robot: An International Journal, 42(5):457-465, 2015.

[23] A. Mohseni-Kabir, C. Rich, S. Chernova, C. L. Sidner, and D. Miller. Interactive hierarchical task learning from a single demonstration. In Proceedings of the Tenth Annual ACM/IEEE International Conference on Human-Robot Interaction, HRI '15, pages 205-212, New York, NY, USA, 2015. ACM.

[24] B. Nemec, R. Vuga, and A. Ude. Efficient sensorimotor learning from multiple demonstrations. Advanced Robotics, 27(13):1023-1031, 2013.

[25] S. Niekum, S. Osentoski, G. Konidaris, S. Chitta, B. Marthi, and A. G. Barto. Learning grounded finite-state representations from unstructured demonstrations. The International Journal of Robotics Research, 34(2):131-157, 2015.

[26] M. Pardowitz, S. Knoop, R. Dillmann, and R. D. Zöllner. Incremental learning of tasks from user demonstrations, past experiences, and vocal comments. IEEE Transactions on Systems, Man, and Cybernetics, Part B (Cybernetics), 37(2):322-332, April 2007.

[27] P. Pastor, M. Kalakrishnan, S. Chitta, E. Theodorou, and S. Schaal. Skill learning and task outcome prediction for manipulation. In 2011 IEEE International Conference on Robotics and Automation, pages 3828-3834, May 2011.

[28] M. R. Pedersen, D. L. Herzog, and V. Krüger. Intuitive skill-level programming of industrial handling tasks on a mobile manipulator. In 2014 IEEE/RSJ International Conference on Intelligent Robots and Systems, pages 4523-4530, Sept 2014.

[29] M. R. Pedersen, L. Nalpantidis, R. S. Andersen, C. Schou, S. Bøgh, V. Krüger, and O. Madsen. Robot skills for manufacturing: From concept to industrial deployment. Robotics and Computer-Integrated Manufacturing, 37:282 - 291, 2016.

[30] A. Perzylo, N. Somani, S. Profanter, I. Kessler, M. Rickert, and A. Knoll. Intuitive instruction of industrial robots: Semantic process descriptions for small lot production. In IEEE/RSJ International Conference on Intelligent Robots and Systems (IROS), Daejeon, Republic of Korea, October 2016. https://youtu.be/bbInEMEF5zU.

[31] G. B. Rodamilans, E. Villani, L. G. Trabasso, W. R. d. Oliveira, and R. Suterio. A comparison of industrial robots interface: force guidance system and teach pendant operation. Industrial Robot: $A n$ International Journal, 43(5):552-562, 2016. 
[32] M. Stenmark, J. Malec, and A. Stolt. From high-level task descriptions to executable robot code. In Intelligent Systems' 2014, pages 189-202. Springer, 2014.

[33] M. Stenmark and P. Nugues. Natural language programming of industrial robots. In Robotics (ISR), 2013 44th International Symposium on, pages 1-5. IEEE, 2013.

[34] M. Stenmark, A. Stolt, E. A. Topp, M. Haage, A. Robertsson, K. Nilsson, and R. Johansson. The GiftWrapper: Programming a Dual-Arm Robot With Lead-through. In ICRA Workshop on Human-Robot Interfaces for Enhanced Physical Interactions, 2016.

[35] M. Stenmark and E. A. Topp. From demonstrations to skills for high-level programming of industrial robots. In AAAI Fall Symposium Series 2016, Symposium on AI for HRI, 2016.

[36] A. Stolt, M. Linderoth, A. Robertsson, and R. Johansson. Force controlled assembly of emergency stop button. In Robotics and Automation (ICRA), 2011 IEEE International Conference on, pages 3751-3756. IEEE, 2011.
[37] A. Stolt, M. Stenmark, A. Robertsson, and K. Nilsson. Robotic gift wrapping or a glance at the present state in santa's workshop. In Reglermote 2016 (Swedish meeting on automatic control 2016), 2016.

[38] P. Tsarouchi, S. Makris, and G. Chryssolouris. Human-robot interaction review and challenges on task planning and programming. Int. J. Comput. Integr. Manuf., 29(8):916-931, Aug. 2016.

[39] M. Tykal, A. Montebelli, and V. Kyrki. Incrementally assisted kinesthetic teaching for programming by demonstration. In 2016 11th ACM/IEEE International Conference on Human-Robot Interaction (HRI), pages 205-212, March 2016.

[40] S. Wrede, C. Emmerich, R. Grünberg, A. Nordmann, A. Swadzba, and J. Steil. A user study on kinesthetic teaching of redundant robots in task and configuration space. Journal of Human-Robot Interaction, 2(1):56 - 81, 2013. 